\title{
INVOLUTIVE SINGULARITIES
}

\author{
By Nguyen HuU DuC
}

\begin{abstract}
Involutive varieties can be found in many problems of differential geometry, calculus of variations, D-module theory and mathematical physics. The aim of this note is to present some problems concerning singularities of germs of involutive varieties and to discuss some recent results in this topic.
\end{abstract}

\section{Involutive Analytic Set}

A symplectic structure on a manifold $M$ is a closed non-degenerate 2 -form $\omega$ and this manifold is called a symplectic manifold.

Darboux theorem says that on a symplectic manifold $M$ there exists a local coordinate system so called canonical coordinate system $\left(p_{1}, . ., p_{n} ; q_{1}, . ., q_{n}\right)$, such that the symplectic structure of $M$ is of the following form

$$
\omega=\sum_{i=1}^{n} d p_{i} \wedge d q_{i} .
$$

Exemple 1. Let $Q$ be a complex analytic manifold. Then its cotangent bundle $T^{*} Q$ is a symplectic manifold with $\omega=d \tilde{\alpha}$, where $\alpha$ is a Liouville form. In the local coordinates $\tilde{\alpha}$ can be written in the form

$$
\tilde{\alpha}=\sum_{i=1}^{n} p_{i} d q_{i},
$$

where $p_{1}, . ., p_{n}$ (resp. $\left.q_{1}, . ., q_{n}\right)$ are coordinates of $Q$ (resp. cotangent coordinates ).

An automorphism of a symplectic manifold $M$ is said to be symplectic if it preserves the symplectic structure of $M$.

Let $f$ be a symplectic automorphism of $T^{*} Q$. We say $f$ is strongly symplectic if $f$ preserves a Liouville 1-form $\tilde{\alpha}$.

Remark. In [W], Weinstein proved that a symplectic automorphism is strongly symplectic iff it is a cotangent map of some automorphism of $Q$.

An automorphism $\Phi$ of $M \times T$ is said to be the deformation of a (strongly) symplectic automorphism $h$ of $M$ if $\Phi(x, t)=\left(h_{t}(x), t\right)$ where $h_{t}$ are (strongly) symplectic

Received August 5, 1993. 
automorphisms of $M$ and $h_{0}=h$.

$A$ contact structure on a manifold $W$ of the dimension $2 n+1$ is a field of kernels of a contact form $\alpha$ on $W$ (i.e. a 1 -form $\alpha$ such that $\alpha \wedge(d \alpha)^{n} \neq 0$ ).

Darboux theorem says that on a contact manifold $W$ there exists a local coordinate system so called Darboux coordinate system $\left(p_{1}, . ., p_{n} ; q_{1}, . ., q_{n} ; z\right)$ such that the contact form is of the form

$$
\alpha=d z-\sum_{i=1}^{n} p_{i} d q_{i}
$$

Exemple 2. Let $Q$ be a complex analytic manifold, let $J^{1}(Q, K)$ be a manifold of 1-jets of holomorphic functions from $Q$ to $C$. This manifold is contact with a natural contact structure, the hyperplanes are defined as the closure of the tangent spaces to the 1-graphs of functions $f: Q \longmapsto C$ (the 1-graph of a function $f$ is the image of the map associating to a point the 1-jet of $f$ at $x)$. Let $\left(p_{1}, . ., p_{n}\right)$ be a local coordinates of $Q,\left(q_{1}, . ., q_{n}\right)$ the values of the first partitial derivatives of the function $z=f(p)$, then

$$
\alpha=d z-\sum_{i=1}^{n} p_{i} d q_{i} .
$$

Let $W$ be a contact manifold with 1-form $\alpha$, we say that an automorphism $G$ of $W$ is contact if $G$ preserves $\alpha$. We can prove that if $G$ is a contact automorphism of $J^{1}(Q, C)$ then $G$ induces a symplectic automorphism h satisfying the following condition:

$$
h^{*}\left(\varrho^{*}(\alpha)\right)=\varrho^{*}(\alpha)+d S .
$$

Where $S$ is a holomorphic function on $T^{*}(Q)$ and $\varrho: T^{*}(Q) \ni(q, p) \longrightarrow(q, p, 0) \epsilon$ $J^{1}(Q, C)$ where $(q, p, z)$ is a Darboux coordinate system of $J^{1}(Q, C)$. Conversely for a given symplectic automorphism $\mathrm{h}$ satisfying the above condition there exists a contact automorphism $G$ that $G$ is a lifting of $h$.

We say that a contact automorphism is strongly contact automorphism if its induced symplectic automorphism is strongly symplectic automorphism. Using Weiestein theorem mentioned in the Remark, we can prove that a germ of contactomorphism $G$ is a germ of strongly contactomorphism iff $G^{*} z n n n n=z$.

An automorphism $G_{T}$ of $W \times T$ is said to be a contact deformation of a (strongly) contact automorphism if it is of the form $G_{T}(x, t)=\left(g_{t}(x), t\right)$, where $g_{t}$ is (strongly) contact automorphism. Of course, each contact deformation induces one symplectic deformation.

Involutive analytic sets:

We say that an analytic subset $\Lambda$ of a symplectic manifold $M$ is involutive if for every point $m$ belonging to an dense subset of the regular part of $\Lambda$ the tangent space $T_{m} \Lambda$ is involutive, this does mean that

$$
\left\{\xi \in T_{m} \Lambda: \omega\left(\xi, T_{m} \Lambda\right)=0\right\} \subset T_{m} \Lambda .
$$

Remark. The condition of $\Lambda$ to be involutive is equvalent to that

$$
\left\{\xi \in T_{m} \Lambda: \omega\left(\xi, T_{m} \Lambda\right)=0\right\} \subset T_{m} \Lambda
$$


for every $m$ belonging to the regular part of $\Lambda$ (see the proof in [B]).

Because a contact manifold of the dimension $2 n+1$ can be considered as a submanifold of the symplectic manifold of the dimension $2 n+2$ with homogeneous coordinates, then we can define involutive set in a contact manifold as an involutive set in homogeneous coordinate system.

We say that an analytic subset $V$ (resp. $\Lambda$ ) of a contact manifold $W$ (resp. a symplectic manifold $M$ ) is legendrian (resp.lagrangian) if it is involutive and it has the pure dimension $n$. We remark that an analytic subset $V$ (resp. $\Lambda$ ) of a contact manifold $W$ (resp. a symplectic manifold $M$ ) is legendrian (resp. lagrangian) if it has the pure dimension and $\alpha \mid V=0$ (resp. $\omega \mid \Lambda=0$ ).

Let $W$ be a germ of a contact manifold with $\alpha$, consider the field of null-spaces (of dimension 1) of $d \alpha$. The segments of the integral curves of this field in a neighbourhood of fixed point under consideration form a manifold $M$ of even dimension. Consider the projection $\bar{\omega}: W \longmapsto M$ along the the integral curves. We may prove that $M$ is symplectic, with a symplectic structure $\omega$ satisfying

$$
d \alpha=-\bar{\omega}^{*}(\omega) .
$$

Because we are interested in the local problems, from now on, we treat $W$ (resp. $M$ ) as $J^{1}(Q, C)$ (resp. $\left.T^{*}(Q)\right)$ for some $Q$ and consider the natural projection:

$$
\bar{\omega}: J^{1}(Q, C) \longmapsto T^{*}(Q),
$$

which sends every $(x, d f(x), f(x))$ to $(x, d f(x))$.

Of course if $V$ is a legendrian germ then $V$ does'nt contain $\bar{\omega}^{-1}(0)$ and then the image of $V i n M$ is lagrangian. Now given is a lagrangian germ in $M$, then it is a projection of only one legendrian germ (because the fiber of $\bar{\omega} \mid V$ over a point $a \in \Lambda$ consists of only one point $\bar{a}$ given in Darboux coordinates as

$$
\left.z(\bar{a})=\int_{0}^{a} \sum_{i=1}^{n} p_{i} d q_{i}\right)
$$

Remark. Every legendrian or lagrangian set is involutive. An analytic set containing an involutive subset is involutive.

We say that an involutive set on $W($ resp. $M$ ) is good if it contains a legendrian set (resp.lagrangian set). By similar argument as above we can prove that there exists one to one correspondence between good involutive germs on $W$ and $M$.

QUESTION. Is it true that there is one-to-one correspondence between involutive germs on $W$ and $M$ ?

We remark that the involutivity property is invariant under symplectic and contact automorphism, hence we can define an equivalence relation between two involutive germs as follows: Two involutive germs are said to be (strongly) equivalent if there exists a (strongly) symplectic or contact automorphism that sends first to second one.

In next section we study two kinds of equivalence relations of involutive germs. 


\section{Involutive Deformations}

From now on, we are interested only in germs of maps, germs of analytic manifolds and germs of analytic sets. For abbreviation, let's us speak of maps, manifolds, and sets, instead of germs of maps, germs of manifolds and germs of sets.

Let $W($ resp. $M)$ be a complex analytic contact (symplectic) manifold, let $T$ be a germ of complex analytic manifold at $t=0$. We say that a germ of analytic subset $V_{T}$ (resp. $\Lambda_{T}$ ) of $W \times T$ (resp. $\left.M \times T\right)$ is an involutive deformation of $V$ (resp. $\Lambda$ ) if

a) $V_{T}\left(\operatorname{resp} . \Lambda_{T}\right)$ is flat over $T$ with respect to the projection $\Pi: W \times T \longmapsto T$ (resp. $\Gamma: M \times T \longmapsto T)$

b) every fiber $V_{t}$ (resp. $\Lambda_{t}$ ) is involutive

c) a special fiber $V_{0}$ (resp. $\left.\Lambda_{0}\right)$ is reduced and coincides with $V$ (resp. $\Lambda$ ).

Remark. By flatness of the condition a) all fibers near 0 have the same dimension. If $V$ is lagrangian or legendrian then fibers near 0 must be lagrangian or legendrian (because involutive analytic set in a symplectic or contact manifold of minimal dimension is lagrangian or legendrian). Hence this definition is a generalization of the definition in [DP].

We say that two deformations $V_{T}$ and $\tilde{V}_{T}$ (resp. $\Lambda_{T}$ and $\tilde{\Lambda}_{T}$ ) are contact (resp. symplectically) equivalent if there is a deformation of contact identity of $W$ (resp. $M$ ) which sends the first to the second one. An involutive germ is said to be contact (resp.symplectically) stable if every deformation is contact (resp. symplectically) equivalent to the constant deformation.

By the similar way we may define a strong equivalence of two deformations and a strong stability of a given lagrangian or legendrian germ.

In next paragraphs we discuss two kinds of equivalence relations between deformations of involutive germs.

\section{Equivalence of involutive deformations}

We say that a germ of involutive analytic set on a contact manifold at a regular point $q$ is degenerate at $q$ if the restriction of Liouville form to this set vanishes at $q$. A deformation is degenerate if all fibers are degenerate at $q$.

In [O], Oshima proved that on the contact manifold

a) Every nondegenerate involutive germ is contact equivalent to the following:

$$
p_{1}=. .=p_{d}=0
$$

b) Every degenerate involutive germ is contact equivalent to the following:

$$
p_{1}=\ldots=p_{d-1}=0, z+h(\hat{p}, \hat{q})=0
$$

Where $\hat{p}=\left(p_{d}, p_{n}\right), \hat{q}=\left(q_{d}, q_{n}\right)$ and $d$ is a codimension of the involutive germ.

On a symplectic case, we have the following:

Proposition 1. On a symplectic manifold every involutive germ is symplectically equivalent following form:

$$
p_{1}=. .=p_{d}=0
$$


In fact, following Oshima's proof we can prove that on a symplectic manifold every involutive germ is symplectically equivalent to one of two following forms:

$$
p_{1}=. .=p_{d}=0
$$

or

$$
p_{1}=\ldots=p_{d-1}=0, p_{d}+h(\hat{p}, \hat{q})=0
$$

But by the Corollary 5.3.31 in [AM] that states as follows: Let $M$ a (complex analytic) symplectic manifold, $\operatorname{dim} M=2 n$, let $f_{1}, \ldots, f_{d}$ be holomorphic functions on $M$. Assume $\left\{f_{\imath}, f_{j}\right\}=0$ and $d f_{1}, \ldots, d f_{d}$ are pointwise linearly independent. Then locally there exist canonical coordinates $\left(P_{1}, \ldots, P_{n}, Q_{1}, \ldots, Q_{n}\right)$ such that $f_{1}=P_{1}, \ldots, f_{d}=P_{d}$.

In our case, if we take $f_{\imath}=p_{i}, i=1, \ldots, d-1, f_{d}=p_{d}+h(\hat{p}, \hat{q})$, the the second case leads to the first case.

Proposition 2. In surtable coordinate system on the contact manifold, each degenerate involutive deformation of degenerate involutive analytic germ can be written in the following form:

$$
p_{1}=\ldots=p_{d-1}=0, z+h(\hat{p}, \hat{q}, t)=0
$$

The proof of this proposition is based on the idea of Oshima by using the Parameter Implicat Function Theorem.

An involutive germ on $M$ (resp. $W$ ) is called regular $r$-cubic configuration if in some canonical coordinate system, this germ is of the form:

$$
p_{1} q_{1}=\ldots=p_{r} q_{r}=p_{r+1}=\ldots=p_{d}=0
$$

(resp.

$$
\left.p_{1} q_{1}=\ldots=p_{r} q_{r}=p_{r+1}=\ldots=p_{d}=0, z=0\right)
$$

This configuration consists of $2^{r}$ irreducible analytic components being smooth involutive germs.

Remark. Above notion is a generalization of [DP] one.

By a similar method in [DP] we get the main result of this section as follows:

THEOREM 3. Every involutıve deformation of regular $r$-cubic configuration is symplectically (resp. contact) equivalent to the following:

$$
p_{1} q_{1}+h_{1}(\hat{p}, \hat{q}, t)=\ldots=p_{r} q_{r}+h_{r}(\hat{p}, \hat{q}, t)=p_{r+1}=\ldots=p_{d}=0
$$

(resp.

$$
\left.p_{1} q_{1}+h_{1}(\hat{p}, \hat{q}, t)=\ldots=p_{r} q_{r}+h_{r}(\hat{p}, \hat{q}, t)=p_{r+1}=\ldots=p_{d}=0, z=0\right)
$$

The proof of this theorem is based on the idea of the proof of theorem 1.2 in [DP] with some modifications.

Remark. Full proofs of Propositions 1, 2 and Theorem 3 can be found in [D]. The above result is a generalization of Theorem 1.2 of [DP] for the involutive case. In the case 
$d=n$, lagrangian deformation of regular $r$-cubic configuration is very simple, equivalent to the following:

$$
p_{1} q_{1}+h_{1}(t)=\ldots=p_{r} q_{r}+h_{r}(t)=p_{r+1}=\ldots=p_{d}=0
$$

and by the corollary of Theorem 1.2 of [DP] every deformation of legendrian regular $r$-cubic configuration is contact-trivial.

In the involutive case, if $r=0$ then every involutive germ of the form

$$
p_{1}=\ldots=p_{r}=p_{r+1}=\ldots=p_{d}=0
$$

or

$$
p_{1}=\ldots=p_{r}=p_{r+1}=\ldots=p_{d}=0, z=0
$$

is symplectically or contact-stable too.

Problem. Characterize involutive deformations of the following germ

$$
p_{1} q_{1}=\ldots=p_{r} q_{r}=p_{r+1}=\ldots=p_{d}=z+h(\hat{p}, \hat{q})=0
$$

on the contact manifold.

\section{Strong equivalence of involutive germs and generating family}

In this section we recall some results in [DDP] and [DP] concerning the characterization of lagrangian and legendrian regular $r$-cubic configuration by phase function.

Let $Z$ be a germ of a smooth (analytic) variety of dimension $m \geqq r$ with a divisor in normal crossing consisting of $r$ components

$$
Z_{1}, . ., Z_{r}
$$

For each $\sigma \subset\{1, . . r\}$ we denote

$$
Z_{\sigma}=\cap_{i \in \sigma} Z_{i}, Z_{\emptyset}=Z
$$

A germ $Z$ with

$$
\underline{Z}=\left(Z_{\sigma}\right)
$$

is called a germ of $r$-reticular variety.

Let $\varphi: Z \longmapsto C$ be a germ of an analytic function, we say that $\varphi$ is $r$-reticular if $Z$ is a germ of $r$-reticular variety and we denote by

$$
\varphi: \underline{Z} \longmapsto C
$$

An automorphism

$$
\phi: Z \longmapsto Z
$$

is called reticular if

$$
\phi\left(Z_{\sigma}\right)=Z_{\sigma}
$$

for every $\sigma$.

Two deformations of a reticular function are said to be $\underline{R}$-equivalent (resp. $\underline{R}^{+}$. equivalent) if there is a reticular automorphism sending first to second one (resp. with a translation). Let

$$
\Psi: Z \times T \longmapsto C
$$


be a deformation of

$$
\varphi: \underline{Z} \longmapsto C
$$

We say that a deformation $\Psi$ of $\varphi$ is $\underline{R}^{+}$(resp. $\underline{R}$ )-versal if every deformation of $\varphi$ is $\underline{R}^{+}$ (resp. $\underline{\mathrm{R}}-$ ) equivalent to the deformation induced by $\Psi$.

Let $\Psi$ be a deformation of a reticular function. A critical set of this deformation is defined as follows:

$$
\begin{gathered}
\Sigma=\cup \Sigma_{\sigma}, \\
\Sigma_{\sigma}=\left\{(q, z) \in Q \times Z:\left.\frac{\partial \Psi}{\partial z}\right|_{z_{\sigma}}=0\right\} .
\end{gathered}
$$

We say that the deformation $\Psi$ satisfies "the reticular nondegeneracy condition" if $\partial_{z} \Psi$ is transversal to every $S_{\sigma}^{1}$ where

$$
\partial_{z} \Psi: Q \times Z \ni(q, z) \longmapsto \partial_{z} \Psi \in T^{*} Z
$$

and

$$
S_{\sigma}^{1}=\left\{\zeta \in T^{*} Z: \zeta \mid Z_{\sigma}=0\right\} .
$$

We see that

$$
\Sigma_{\sigma}=\left(\partial_{z} \Psi\right)^{-1}\left(S_{\sigma}^{1}\right) .
$$

Let $\chi$ be a characteristic map defined as follows:

$$
\chi: \Sigma \longrightarrow J^{1}(Q, C)
$$

which sends $(q, z)$ to 1 -jet of $\Psi$ with $z$ constant, and let

$$
\chi_{\sigma}=\chi \mid \Sigma_{\sigma},
$$

We can prove that $\chi_{\sigma}$ is a legendrian immersion and

$$
V(\Psi)=\left\{\operatorname{Im}\left(\chi_{\sigma}\right)\right\}
$$

form a regular $r$-cubic configuration.

By the same way we can define another characteristic map

$$
\tilde{\chi}: \Sigma \longrightarrow T^{*} Q \text {. }
$$

We can prove that

$$
\tilde{\chi}_{\sigma}=\tilde{\chi} \mid \Sigma_{\sigma}
$$

are lagrangian immersions and

$$
\Lambda(\Psi)=\left\{\operatorname{Im}\left(\tilde{\chi}_{\sigma}\right)\right\}
$$

form a regular $r$-cubic configuration.

The deformation $\Psi$ satisfying the reticular nondegeneracy condition is called a generating family for regular $r$-cubic configuration and $V(\Psi)$ (resp. $\Lambda(\Psi)$ ) is called the characteristic variety on $J^{1}(Q, C)$ (resp. on $T^{*}(Q)$ ) of $\Psi$. Now we may formulate main results of this subsection.

THEOREM 4. Let $V$ be a regular $r$-cubic configuration, such that $\left.\Pi\right|_{V}$ is finite, where $\Pi$ is a projection of the bundle $J^{1}(Q, C)$, then there exists a generating family $\Psi$ such that $V(\Psi)=V$. 
THEOREM 5. Let $\Psi_{1}$ and $\Psi_{2}$ be generating families with the same base $Q$, then

$$
V\left(\Psi_{1}\right)=V\left(\Psi_{2}\right) \Longleftrightarrow \Psi_{1} \cong \Psi_{2},
$$

where $\cong$ means a $\underline{R}$-equivalence.

THEOREM 6. $V(\Psi)$ is stable, if and only if $\Psi$ is $\underline{\mathrm{R}}^{+}$-versal.

Remark. Theorems 4,5 and 6 are still true for $\Lambda$ on $T^{*} Q$. Theorem 4 is proved in [DP] by using the existence of generating function of the canonical transformation. We remark that, in [DDP] we have proved analogous theorem, but the method is very complicated, because the notion of regular $r$-cubic configuration introduced in [DDP] is very weak. Theorem 5 is proved in [DDP]. The proof of Theorem 6 is given in [DP]. In the case $r=1$, these theorems are proved in [DD]. In the case $r=0$, they are proved by Zakalyukin (see [Z]).

Generatıng family for involutıve germ

We see that for each germ of lagrangian or legendrian variety being regular $r$-cubic configuration we can construct generating family uniquely determined in the sense of Theorem 5. Therefore we hope that for every germ of involutive variety being regular $r$-cubic configuration, we can construct a generating family.

We can consider an involutive germ as a family of germs of lagrangian varieties. In fact, If $\Lambda$ is involutive set in $M$ then the its complementary part by the symplectic form $\omega$ form an involutive distribution. Maximal connected integral manifolds of $D$ are called characteristics. Let $Y$ be the set of characteristics of $\Lambda$ and $\delta: \Lambda \longrightarrow Y$ be a projection along characteristics. Then the graph of this projection is a lagrangian variety of $M \times Y$ (see [AM]). By similar argument we can consider an involutive set on the contact manifold as a family of germs of legendrian varieties. Therefore from above fact we can prove that any involutive germ on $W$ is a subset of some legendrian germ. In [J] Janeczko constructed a generating family for a germ of involutive submanifold on symplectic manifold by constructing a generating family for above graph (because $\Lambda$ is smooth therefore the graph is smooth too). We can also construct a generating family for-a germ of involutive submanifold of a contact manifold.

Problem. 1. Find an equivalence relation that two generating family defining the same germ of involutive submanifold are equivalent.

2. Is it possible to contruct a generating family for an involutive regular $r$-cubic configuration?

We hope that we can resolve a part 2 of the problem by combining the last construction of lagrangian variety and analogous method of constructing a generating family for lagrangian regular $r$-cubic configuration.

\section{REFERENCES}

[AG] V.I. Arnold and A.B. Givental, Symplectic Geometry. Encyclopaedia of Mathematical Sciences, vol. 4, Springer-Verlag 1990.

[AM] R. Abraham and J.E. Marsden, Foundations of Mechanics. Addison-Wesley Publishing Com- 
pany, Inc. 1987.

[B] J.E. Björk, Analytic D-modules and applications. Kluwer Academic Publishers, 1993.

[D] N.H. Duc, Involutive singularities. In preparation.

[DD] N.H. Duc and N.T. Dai, Stabilité de l'interaction géometrique entre deux composantes simples holonomes. C.R. Acad. Sci. Paris $t 291$ (15 Septembre 1980) Serie A pp. 113-116.

[DDP] N.T. Dai, N.H. Duc and F. Pham, Singularités nondégénérées des systèmes de Gauss-Manin réticulés. Nouvelle Serie de Bulletin de la Societé Math. de France, no. 6, 1981.

[DP] N.H. Duc and F. Pham, Configurations legendriannes stables et fonctions d́Airy-Weber generalise'es. Ann. Inst. Fourier, Grenoble, 41, 4 (1991), 905-936.

[J] S. Janeczko, Coisotropic varieties and their generating families. Ann. Inst. Heri Poincaré, Vol. 56, n. 4, 1992, pp. 429-441.

[O] T. Oshima, Singularities in contact geometry and degenerate pseudo-differential equations. J. Fac. Sci. Univ. Tokyo, IA, 21 (1974), 43-83.

[W] A. Weinstein, Lectures on symplectic manifolds. C.B.M.S. Conf. Series., Am. Math. Soc.., no. 29. A.M.S. Providence 1977.

[Z] V.M. Zakalyukin, Singularities of lagrangian and legendrian submanifolds. Funk. An. and Pril. 10 (1), 1976, pp. 26-36.

DEPARTMENT OF MATHEMATICS

UNIVERSITY OF DALAT

DALAT, ViETnAM 